\title{
Bile Granuloma Mimicking Peritoneal Seeding: A Case Report
}

\author{
Hasong Jeong · Hye Won Lee \\ Hye Ra Jung · Ilseon Hwang \\ Sun Young Kwon · Yu Na Kang \\ Sang Pyo Kim · Misun Choe
}

Department of Pathology, Keimyung University

School of Medicine, Daegu, Korea

Received: March 30, 2018

Revised: May 28, 2018

Accepted: June 2, 2018

\section{Corresponding Author}

Misun Choe, MD

Department of Pathology, Keimyung University

School of Medicine, 1095 Dalgubeol-daero,

Dalseo-gu, Daegu 42601, Korea

Tel: +82-53-580-3815

Fax: +82-53-580-3823

E-mail:msc@dsmc.or.k

\begin{abstract}
Laparoscopic cholecystectomy is a widely used treatment method for most cholelithiasis and is a relatively safe procedure. Foreign body granulomatous reaction to bile or gallstone spillage during laparoscopic cholecystectomy has rarely been reported. We report a case of bile granuloma after laparoscopic cholecystectomy, which mimicked peritoneal seeding. A 59-year-old Korean man presented with right upper quadrant pain. He underwent laparoscopic cholecystectomy for acute cholecystitis with cholelithiasis. Pathologic examination revealed an incidental adenocarcinoma invading the lamina propria with acute cholecystitis and cholelithiasis. After 3 months, follow-up abdominal computed tomography revealed a subhepatic nodule, which showed hypermetabolism on positron emission tomography-computed tomography. Suspecting localized peritoneal seeding, wedge resection of the liver, wedge resection of the transverse colon, and omentectomy were performed. Pathologic examination of the resected specimens revealed multiple bile granulomas. Awareness of bile granuloma mimicking malignancy is noteworthy for patient management to reduce unnecessary procedure during postoperative surveillance.
\end{abstract}

Key Words: Bile; Granuloma; Cholecystectomy
Laparoscopic cholecystectomy is a widely used treatment method for acute cholecystitis that has many advantages over open cholecystectomy. ${ }^{1}$ Complications following laparoscopic cholecystectomy are relatively insignificant; however, bile or gallstone spillage during laparoscopic cholecystectomy is not infrequent, occurring in $10 \%$ to $40 \%$ of cases. ${ }^{2}$ In most cases, they generally have no detrimental consequences, but might lead to peritoneal granulomatous inflammation. ${ }^{3-6}$ The differential diagnoses include various infectious and noninfectious causes of peritoneal granuloma and may clinically result in misdiagnosis as malignancy. ${ }^{4}$ Here, we report a case of bile granuloma that mimicked peritoneal seeding following laparoscopic cholecystectomy.

\section{CASE REPORT}

A 59-year-old Korean man presented with right upper quadrant pain for 3 days. He had no previous medical history. Physical examination was unremarkable except for right upper quadrant tenderness. C-reactive protein level was $15.89 \mathrm{mg} / \mathrm{dL}$ (normal $<0.5 \mathrm{mg} / \mathrm{dL})$. Total bilirubin $(1.42 \mathrm{mg} / \mathrm{dL}$; normal, $<1.2 \mathrm{mg} /$ $\mathrm{dL})$ and direct bilirubin $(1.18 \mathrm{mg} / \mathrm{dL}$; normal, $<0.4 \mathrm{mg} / \mathrm{dL})$ were slightly increased. Other laboratory findings, including carcinoembryonic antigen (CEA), CA19-9, liver enzyme, and kidney function test results, were within normal ranges. Abdominal computed tomography $(\mathrm{CT})$ revealed a markedly distended gallbladder and a 32-mm polypoid mass in the neck of the gallbladder, suggesting acute calculous cholecystitis or gallbladder cancer. The patient underwent laparoscopic cholecystectomy. During surgery, gallbladder aspiration was performed due to distension. There was no perforation during gallbladder bed dissection with no gross bile contamination of the peritoneum or any conspicuous bile leakage. Gross examination revealed a $2.8 \times 1.6 \mathrm{~cm}$ polypoid mass with several black stones up to $0.9 \mathrm{~cm}$ without perforation. Microscopic findings of the gallbladder showed adenocarcinoma and acute cholecystitis. The adenocarcinoma was limited to the polyp and invaded the lamina propria without lymphovascular invasion, perineural invasion, or involvement of the resection margin.

After 3 months, he had no clinical symptoms, such as abdominal pain or discomfort. Follow-up abdominal CT revealed an ill-defined mass lesion in the right subhepatic space, which showed hypermetabolism on positron emission tomography-computed tomography (PET-CT) (Fig. 1). Follow-up tumor markers were 
within the normal range. Suspecting localized peritoneal seeding, exploratory laparotomy was performed. It revealed adhesion between the liver, omentum, transverse colon, and peritoneum. There were seeding mass-like lesions in the gallbladder bed and adhesion sites. Wedge resection of the liver, wedge resection of the transverse colon, and omentectomy were done. Gross finding of the resected specimens showed ill-defined yellow to brownish nodular lesions up to $1.1 \times 1.0 \mathrm{~cm}$ in the liver and transverse colon without necrosis (Fig. 2). Microscopic findings of the liver, transverse colon, and omentum showed brown pigments and foreign-body type multinucleated giant cells with numerous lymphocytes, suggesting the diagnosis of granulomatous inflammation (Fig. 3). The brown pigment was compatible with bile pigment and was greenish brown on Fouchet's staining. There was no evidence of malignant tumor cells, and stone and crystal deposits were not present. He had no complication or recurrence for 9 months after surgery. This study was approved by the Institutional Review Board of Keimyung University Dongsan Medical Center (IRB No. 2018-03-052) and informed consent was waived.

\section{DISCUSSION}

Although laparoscopic cholecystectomy has many advantages over standard open cholecystectomy, several intraoperative complications of laparoscopic cholecystectomy should be noted. Common complications are iatrogenic perforation of the gallbladder,
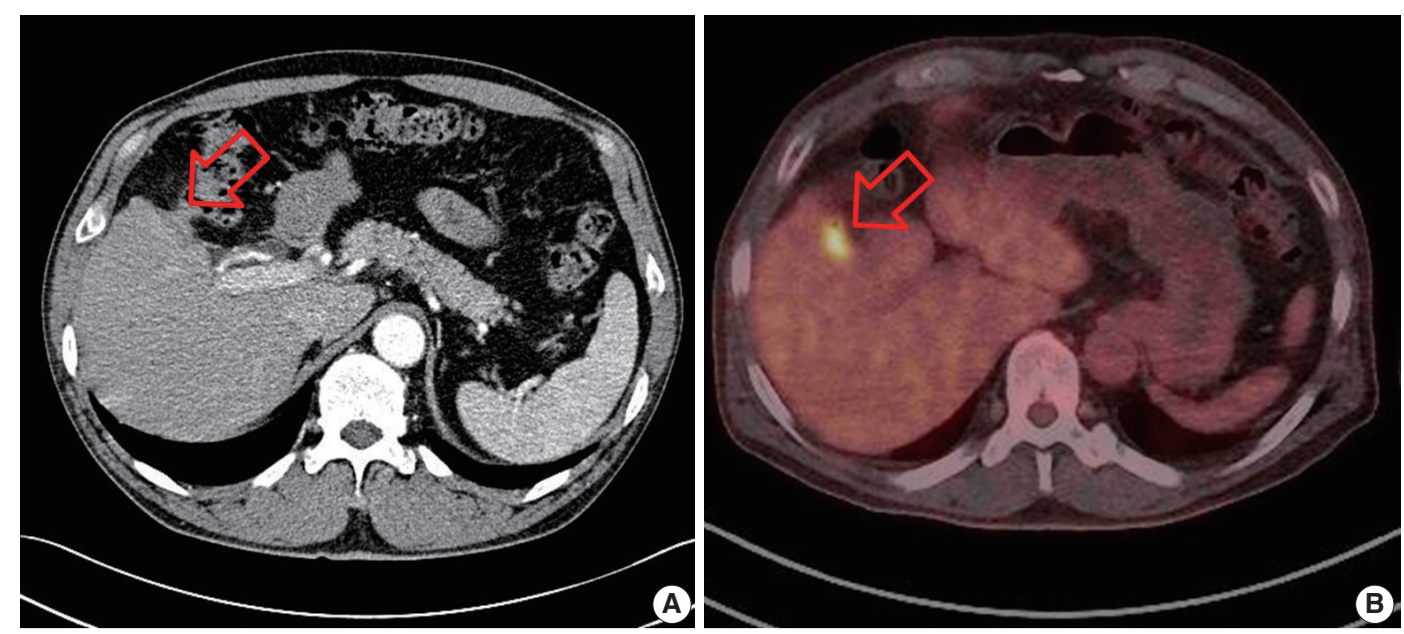

Fig. 1. Findings of abdominal computed tomography $(C T)(A)$ and positron emission tomography-computed tomography $(P E T-C T)(B)$. Abdominal CT showed ill-defined mass lesions (arrow) in the right subhepatic space near the gallbladder bed. PET-CT demonstrated a focal hypermetabolic lesion (arrow) in the right subhepatic space.
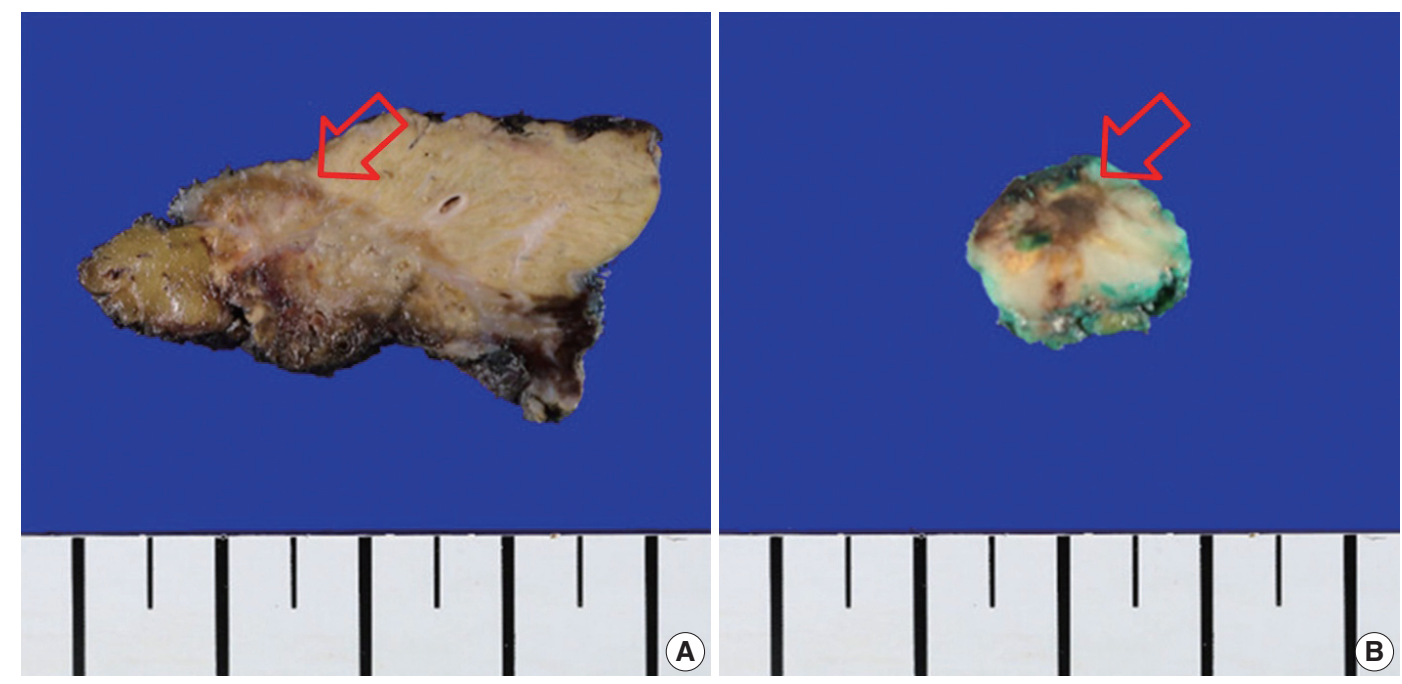

Fig. 2. Gross finding of the specimens showed ill-defined yellow to brownish, variegated, nodular lesions (arrows) without necrosis in the liver (A) and the transverse colon (B). 

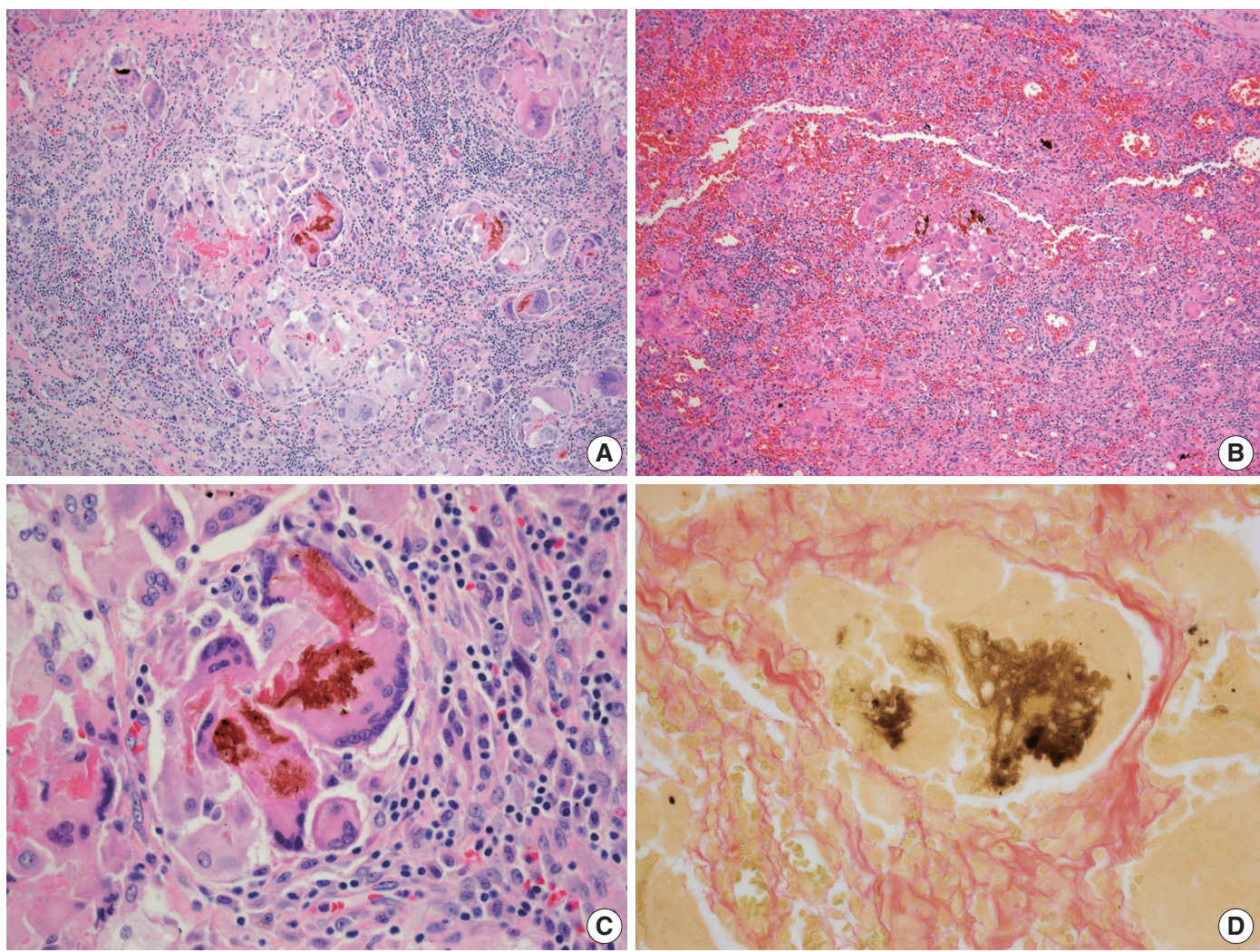

Fig. 3. Microscopic findings of the lesion. The liver (A) and transverse colon (B) showed granulomatous inflammation, which consists of brown pigments and multinucleated giant cells with numerous lymphocytes (C). The pigment was greenish brown on Fouchet's technique (D).

bleeding of the tissues near the gallbladder, and gallstone spillage into the peritoneal cavity. ${ }^{6}$ Bile or gallstone spillage during laparoscopic cholecystectomy is not infrequent, occurring in $10 \%$ to $40 \%$ of cases. ${ }^{2,7}$ Gallbladder aspiration is a technique to reduce the possibility of unintentional perforation during dissection by decreasing the gallbladder volume. It is controversial whether gallbladder aspiration has the effect of reducing operative time or decreasing the risk of perforation. ${ }^{8-10}$ In our patient, gallbladder aspiration was performed to reduce the volume. If there was no perforation or grossly obvious bile leakage during gallbladder bed dissection, a possibility of bile leakage through the aspiration site may be considered. Occurrence of gallstone spillage complications can range from a few days to several years. ${ }^{11}$ Early complication of bile or stone spillage is peritonitis with acute symptoms. Acute symptoms may not occur if bile leakage is mild, or if the surgeon executes immediate irrigation or stone removal. If bile or stone spillage is not accompanied by acute symptoms, it is a silent event without detrimental sequelae. ${ }^{5}$ However, abscess formation and fistula formation are medium- to long-term complications. ${ }^{12}$ Patients with gallstone spillage may be asymptomatic until complications develop. In our patient, if there was no follow- up abdominal CT, he might not have known about the presence of bile granuloma because he had no symptoms.

Foreign body reaction to bile or gallstone spillage during laparoscopic cholecystectomy as the cause of intraperitoneal granulomas has been rarely reported. ${ }^{2,3,5,7,12,13}$ Foreign-body granulomas are generally asymptomatic. ${ }^{14}$ Given that most patients do not undergo follow-up abdominal imaging study after laparoscopic cholecystectomy, they may not know about granulomas if they are asymptomatic. Most cases were found during cesarean section or gynecological surgery. ${ }^{3,5,13}$ Here, we describe a case of multiple bile granulomas with clinical suspicion of peritoneal seeding after laparoscopic cholecystectomy. To our knowledge, three cases similar to our case have been reported. In the first case, a 37-year-old woman who underwent cholecystectomy due to perforated gallbladder showed multiple bile granulomas that mimicked disseminated malignancy during cesarean section. ${ }^{5}$ In the second case, a 59-year-old man presented with foreign body granuloma due to spilled gallstones after laparoscopic cholecystectomy that mimicked a retroperitoneal sarcoma. ${ }^{2}$ In the third case, a 29-yearold woman who underwent laparoscopic cholecystectomy with stone spillage during procedure showed several hard nodules in 
omentum that mimicked metastases from unknown primary tumor during cesarean section. ${ }^{13}$

18-Fluoro-2-deoxyglucose (FDG) is well known to accumulate in inflammatory cells, including neutrophils, lymphocytes, and macrophages due to elevated glucose requirements in various inflammatory conditions. ${ }^{15}$ Therefore, PET-CT may show falsepositive FDG uptake in several benign conditions, including granulomatous disease. Furthermore, foreign body granulomas have been reported to masquerade as malignant tumors. ${ }^{16,17}$ In our case, an increased FDG uptake of the ill-defined mass lesion in the right subhepatic space was mistaken for localized peritoneal seeding.

The current case emphasizes four significant features. Granuloma due to spilled bile or gallstone is a rare complication following laparoscopic cholecystectomy and should be considered in a patient presenting with a peritoneal mass after laparoscopic cholecystectomy. Secondly, the aspiration itself may cause bile leakage without obvious perforation. Thirdly, the surgeon should make a note in the patient's medical record after laparoscopic cholecystectomy whether the gallbladder had been perforated and whether there had been any bile or stone leakage during the procedure for future reference. Finally, a biopsy or diagnostic laparoscopy with frozen section of the peritoneal nodule is preferred over open exploratory laparotomy in questionable cases.

In summary, bile leakage during laparoscopic cholecystectomy could lead to bile granuloma mimicking peritoneal seeding. This reminds the importance of caution when removing the gallbladder in case of acute calculous cholecystitis. In addition, the possibility of bile granuloma should be considered in patients with gallbladder cancer during follow-up after surgery.

\section{ORCID}

Hasong Jeong: https://orcid.org/0000-0002-2205-9586

Hye Won Lee: https://orcid.org/0000-0001-8540-524X

Ilseon Hwang: https://orcid.org/0000-0002-6122-4417

Sun Young Kwon: https://orcid.org/0000-0002-8410-0185

Yu Na Kang: https://orcid.org/0000-0002-5536-8828

Sang Pyo Kim: https://orcid.org/0000-0003-0948-2408

Misun Choe: https://orcid.org/0000-0002-1412-5360

\section{Conflicts of Interest}

No potential conflict of interest relevant to this article was reported.

\section{REFERENCES}

1. Townsend CM, Beauchamp RD, Evers BM, Mattox K. Sabiston textbook of surgery. 20th ed. Philadelphia: Saunders Elsevier, 2017; 1493-500.

2. Kim BS, Joo SH, Kim HC. Spilled gallstones mimicking a retroperitoneal sarcoma following laparoscopic cholecystectomy. World J Gastroenterol 2016; 22: 4421-6.

3. Merchant SH, Haghir S, Gordon GB. Granulomatous peritonitis after laparoscopic cholecystectomy mimicking pelvic endometriosis. Obstet Gynecol 2000; 96 (5 Pt 2): 830-1.

4. Famularo G, Remotti D, Galluzzo M, Gasbarrone L. Granulomatous peritonitis after laparoscopic cholecystectomy. JSLS 2012; 16: 481-4.

5. McVeigh G, McComiskey M, McCluggage WG. Peritoneal bile granulomas identified at Cesarean section and mimicking disseminated malignancy. Int J Surg Pathol 2012; 20: 89-91.

6. Radunovic M, Lazovic R, Popovic N, et al. Complications of laparoscopic cholecystectomy: our experience from a retrospective analysis. Open Access Maced J Med Sci 2016; 4: 641-6.

7. Lee W, Kwon J. Fate of lost gallstones during laparoscopic cholecystectomy. Korean J Hepatobiliary Pancreat Surg 2013; 17: 66-9.

8. Calik A, Topaloglu S, Topcu S, Turkyilmaz S, Kucuktulu U, Piskin B. Routine intraoperative aspiration of gallbladder during laparoscopic cholecystectomy. Surg Endosc 2007; 21: 1578-81.

9. Ezer A, Nursal TZ, Colakoglu T, Noyan T, Moray G, Haberal M. The impact of gallbladder aspiration during elective laparoscopic cholecystectomy: a prospective randomized study. Am J Surg 2008; 196: 456-9.

10. Siddiqui MR, Sajid MS, Nisar A, Ali H, Zaborszky A, Hasan F. A meta-analysis of outcomes after routine aspiration of the gallbladder during cholecystectomy. Int Surg 2011; 96: 21-7.

11. Pazouki A, Abdollahi A, Mehrabi Bahar M, et al. Evaluation of the incidence of complications of lost gallstones during laparoscopic cholecystectomy. Surg Laparosc Endosc Percutan Tech 2014; 24: 213-5.

12. Zehetner J, Shamiyeh A, Wayand W. Lost gallstones in laparoscopic cholecystectomy: all possible complications. Am J Surg 2007; 193: 73-8.

13. Suarez-Zamora DA, Barrera-Herrera LE, Caceres-Mileo R, PalauLazaro MA. Intraperitoneal granulomas unexpectedly found during a Cesarean delivery: a late complication of dropped gallstones. Case Rep Pathol 2017; 2017: 4873273.

14. Luo J, Mao Y, Cai S, Shen X, Chen S, Xie L. Post-nephrectomy foreign-body granuloma in the retroperitoneum mimicking lymph node metastasis of renal cell cancer. Onco Targets Ther 2014; 7: 
2137-41.

15. Pantiora EV, Kontis EA, Michalaki V, et al. Granuloma mimicking local recurrence on PET/CT after liver resection of colorectal liver metastasis: a case report. Cureus 2016; 8: e717.

16. Erdoğan D, Bozkurt C, Özmen Ö, Boduroglu E, Sahin G. Foreign body reaction with high standard uptake value level in 18-FDG PET/CT mimicking relapse in an 8-year-old patient diagnosed with Hodgkin lymphoma: a case report. European J Pediatr Surg Rep 2013; 1: 60-2.

17. Kim SW, Shin HC, Kim IY, Baek MJ, Cho HD. Foreign body granulomas simulating recurrent tumors in patients following colorectal surgery for carcinoma: a report of two cases. Korean J Radiol 2009; 10: 313-8. 